\title{
Polynomial Imaginary Decompositions for Finite Separable Extensions
}

by

\author{
Adam GRYGIEL
}

\author{
Presented by Andrzej SCHINZEL
}

Summary. Let $K$ be a field and let $L=K[\xi]$ be a finite field extension of $K$ of degree $m>1$. If $f \in L[Z]$ is a polynomial, then there exist unique polynomials $u_{0}, \ldots, u_{m-1} \in$ $K\left[X_{0}, \ldots, X_{m-1}\right]$ such that $f\left(\sum_{j=0}^{m-1} \xi^{j} X_{j}\right)=\sum_{j=0}^{m-1} \xi^{j} u_{j}$. A. Nowicki and S. Spodzieja proved that, if $K$ is a field of characteristic zero and $f \neq 0$, then $u_{0}, \ldots, u_{m-1}$ have no common divisor in $K\left[X_{0}, \ldots, X_{m-1}\right]$ of positive degree. We extend this result to the case when $L$ is a separable extension of a field $K$ of arbitrary characteristic. We also show that the same is true for a formal power series in several variables.

1. Introduction. Throughout the paper, $K$ is a field and $L=K[\xi]$ is a finite field extension of $K$ of degree $m>1$. For $j=1, \ldots, n$ let $\mathbf{X}_{j}=$ $\left(X_{j, 0}, \ldots, X_{j, m-1}\right)$ denote a system of variables and set

$$
\left[\mathbf{X}_{j}\right]=X_{j, 0}+\xi X_{j, 1}+\cdots+\xi^{m-1} X_{j, m-1} .
$$

If $n=1$, then we write briefly $\mathbf{X}=\left(X_{0}, \ldots, X_{m-1}\right)$ instead of $\mathbf{X}_{1}=$ $\left(X_{1,0}, \ldots, X_{1, m-1}\right)$. If $f \in L\left[Z_{1}, \ldots, Z_{n}\right]$ is a polynomial, then there exist unique polynomials $u_{0}, \ldots, u_{m-1} \in K\left[\mathbf{X}_{1}, \ldots, \mathbf{X}_{n}\right]$ such that

$$
f\left(\left[\mathbf{X}_{1}\right], \ldots,\left[\mathbf{X}_{n}\right]\right)=u_{0}+\xi u_{1}+\cdots+\xi^{m-1} u_{m-1} .
$$

This representation is called the imaginary decomposition of $f$ relative to $\xi$, and the polynomials $u_{0}, \ldots, u_{m-1}$ are the imaginary parts of $f$ (see [1]).

Assume that

$$
\phi(t)=t^{m}-a_{m-1} t^{m-1}-\cdots-a_{1} t-a_{0}, \quad \text { where } a_{0}, \ldots, a_{m-1} \in K,
$$

is the minimal polynomial of $\xi$ over $K$ and let $u=\left(u_{0}, \ldots, u_{m-1}\right)$ be a sequence of polynomials belonging to $K[\mathbf{X}]$. Denote by $\bar{u}=\left(\bar{u}_{0}, \ldots, \bar{u}_{m-1}\right)$

2000 Mathematics Subject Classification: Primary 12E05, 12F10.

Key words and phrases: polynomial, decomposition, separable extension. 
the sequence of polynomials defined by

$$
\bar{u}_{0}=a_{0} u_{m-1}, \quad \bar{u}_{1}=a_{1} u_{m-1}+u_{0}, \ldots, \bar{u}_{m-1}=a_{m-1} u_{m-1}+u_{m-2} .
$$

We say that $u$ is a $\xi$-sequence if $u$ satisfies the following generalized CauchyRiemann equations introduced in [1]:

$$
\frac{\partial u}{\partial X_{i}}=\frac{\partial \bar{u}}{\partial X_{i-1}}, \quad i=1, \ldots, m-1 .
$$

In 2003, A. Nowicki and S. Spodzieja proved the following theorem.

TheOREM 1 ([1, Theorem 3.8]). Let $K$ be a field of characteristic zero and let $L=K[\xi]$ be a finite field extension of $K$ of degree $m>1$. The following two conditions are equivalent:

(i) $u$ is a $\xi$-sequence.

(ii) There exists $f \in L[Z]$ such that $u_{0}, \ldots, u_{m-1}$ are the imaginary parts of $f$.

As a consequence of Theorem 1, A. Nowicki and S. Spodzieja also proved the following curious theorem.

TheOREm 2 ([1, Theorem 5.3]). If under the assumptions of Theorem 1 , $u_{0}, \ldots, u_{m-1}$ are the imaginary parts of $f \in L\left[Z_{1}, \ldots, Z_{n}\right] \backslash\{0\}$, then $\operatorname{gcd}\left(u_{0}, \ldots, u_{m-1}\right)=1$.

The assumption that char $K=0$ played an essential role in the proof of Theorem 2. The aim of this paper is to extend this theorem to the case when $L$ is a separable extension of a field $K$ of arbitrary characteristic. More precisely, our main result is the following.

Theorem 3. Let $K$ be a field and let $L=K[\xi]$ be a finite separable extension of $K$ of degree $m>1$. If $u_{0}, \ldots, u_{m-1}$ are the imaginary parts of $f \in L\left[Z_{1}, \ldots, Z_{n}\right] \backslash\{0\}$, then $\operatorname{gcd}\left(u_{0}, \ldots, u_{m-1}\right)=1$.

Additionally, in Section 4 we generalize Theorems 1-3 to formal power series (Propositions 4-6, respectively).

2. Some auxiliary results. To prove Theorem 3 we need several known simple facts (see [1]).

Proposition 1. If $u_{0}, \ldots, u_{m-1}$ are the imaginary parts of a homogeneous polynomial $f \in L\left[Z_{1}, \ldots, Z_{n}\right]$ of degree $s$, then $u_{i}$ is zero or a homogeneous polynomial of degree $s$ for $i=0, \ldots, m-1$.

Proposition 2. If the polynomials $u_{0}, \ldots, u_{m-1} \in K\left[\mathbf{X}_{1}, \ldots, \mathbf{X}_{n}\right]$ are not relatively prime, then their homogeneous components of the highest degree are also not relatively prime. 
Let $d, n \in \mathbb{Z}, d, n \geq 2$. Consider the Kronecker substitution (cf. [2, 1.6, Definition 5]), i.e. the $L$-automorphism $\kappa_{d}$ of $L\left[Z_{1}, \ldots, Z_{n}\right]$ defined by

$$
\kappa_{d}\left(Z_{j}\right)= \begin{cases}Z_{1} & \text { if } j=1, \\ Z_{j}+Z_{1}^{d^{j-1}} & \text { if } j=2, \ldots, n .\end{cases}
$$

Proposition 3 ([1, Proposition 5.1]). Let $f \in L\left[Z_{1}, \ldots, Z_{n}\right]$, and let $d>\max _{j=1, \ldots, n} \operatorname{deg}_{Z_{j}} f>0$. Then

$\kappa_{d}(f)=a Z_{1}^{N}+$ terms of degrees lower than $N, \quad N \geq 1, a \in L \backslash\{0\}$.

Let $P_{j}=\kappa_{d}\left(Z_{j}\right) \in L\left[Z_{1}, \ldots, Z_{n}\right]$ for $j=1, \ldots, n$ and $P_{j}\left(\left[\mathbf{X}_{1}\right], \ldots,\left[\mathbf{X}_{n}\right]\right)=v_{j, 0}+\xi v_{j, 1}+\cdots+\xi^{m-1} v_{j, m-1}, \quad v_{j, i} \in K\left[\mathbf{X}_{1}, \ldots, \mathbf{X}_{n}\right]$. Let $\gamma: K\left[\mathbf{X}_{1}, \ldots, \mathbf{X}_{n}\right] \rightarrow K\left[\mathbf{X}_{1}, \ldots, \mathbf{X}_{n}\right]$ be the homomorphism such that $\gamma\left(X_{j, i}\right)=v_{j, i}$.

Lemma 1 ([1, Lemma 5.2]). $\gamma$ is a $K$-automorphism of $K\left[\mathbf{X}_{1}, \ldots, \mathbf{X}_{n}\right]$.

3. Proof of Theorem 3. A crucial role in the proof is played by the following lemma.

LEMma 2. If under the assumptions of Theorem $3, u_{0}, \ldots, u_{m-1}$ are the imaginary parts of $f(Z)=a_{0} Z^{s}, a_{0} \in L \backslash\{0\}$, then $\operatorname{gcd}\left(u_{0}, \ldots, u_{m-1}\right)=1$.

Proof. Let $\phi$ be the minimal polynomial of $\xi$ over $K$ and let $M$ be a decomposition field of $\phi$. Then $K[\xi]=K(\xi) \subset M$ and $\operatorname{deg} \phi=m>1$. Consequently, since $\xi$ is a simple root of $\phi$, there exists $b \in M, b \neq \xi$, such that $\phi(b)=0$. There is a $K$-isomorphism $\varphi: K(\xi) \rightarrow K(b)$ such that $\varphi(\xi)=b$.

Suppose that there is a polynomial $v \in K[\mathbf{X}]$ of positive degree which is a common divisor of $u_{0}, \ldots, u_{m-1}$ in $K[\mathbf{X}]$, and so also in $L[\mathbf{X}]$. Since $L[\mathbf{X}]$ is a UFD and $X_{0}+\xi X_{1}+\cdots+\xi^{m-1} X_{m-1}$ is irreducible in $L[\mathbf{X}]$, there exist $l \in \mathbb{Z}, l \geq 1$, and $a \in L \backslash\{0\}$ such that

$$
v\left(X_{0}, \ldots, X_{m-1}\right)=a\left(X_{0}+\xi X_{1}+\cdots+\xi^{m-1} X_{m-1}\right)^{l} .
$$

Then $v(-\xi, 1,0, \ldots, 0)=0$, and so, since $v \in K[\mathbf{X}]$, we get

$$
a(-b+\xi)^{l}=v(-b, 1,0, \ldots, 0)=\varphi(v(-\xi, 1,0, \ldots, 0))=0,
$$

a contradiction.

Using the facts in Section 2 we will extend Lemma 2 so as to obtain Theorem 3.

Proof of Theorem 3. Suppose that $u_{0}, \ldots, u_{m-1}$ have a common divisor in $K\left[\mathbf{X}_{1}, \ldots, \mathbf{X}_{n}\right]$ of positive degree. Denote by $f^{(s)}$ the homogeneous part of the highest degree of $f$ and let $u_{0}^{(s)}, \ldots, u_{m-1}^{(s)}$ be the homogeneous parts of the highest degree of $u_{0}, \ldots, u_{m-1}$, respectively. By Proposition 3 and Lemma 1 one can assume that $f^{(s)}\left(Z_{1}, \ldots, Z_{n}\right)=a_{0} Z_{1}^{s}, a_{0} \in L \backslash\{0\}$, and 
so $f^{(s)} \in L\left[Z_{1}\right]$. By Propositions 1 and $2, u_{0}^{(s)}, \ldots, u_{m-1}^{(s)}$ are the imaginary parts of $f^{(s)}$ and they are not relatively prime. This contradicts Lemma 2 and ends the proof.

The following example, due to the referee, shows that the assumption of Theorem 3 concerning separability of the extension $L$ of $K$ is necessary.

Example 1. Let $K=\mathbb{F}_{2}\left(t^{2}\right), L=\mathbb{F}_{2}(t)$ and let $\xi=t$. Consider the polynomial $f(Z)=Z^{2}$. Then

$$
f\left(X_{0}+\xi X_{1}\right)=X_{0}^{2}+t^{2} X_{1}^{2} \in K\left[X_{0}, X_{1}\right] .
$$

Hence $u_{0}=X_{0}^{2}+t^{2} X_{1}^{2}$ and $u_{1}=0$ are the imaginary parts of $f$ and they are not relatively prime.

4. Generalizations to formal power series. In this section we generalize Theorems $1-3$ to formal power series.

Let $f \in L\left[\left[Z_{1}, \ldots, Z_{n}\right]\right]$ be a formal power series of the form $f=\sum_{r=d}^{\infty} f^{(r)}$, where $f^{(r)}$ is zero or a homogeneous polynomial of degree $r$ for $r \geq d$, and let $u_{0}, \ldots, u_{m-1} \in K\left[\left[\mathbf{X}_{1}, \ldots, \mathbf{X}_{n}\right]\right]$ be formal power series of the form $u_{j}=\sum_{r=d}^{\infty} u_{j}^{(r)}$, where $u_{j}^{(r)}$ is zero or a homogeneous polynomial of degree $r$ for $r \geq d, j=0, \ldots, m-1$. By Proposition 1 we get immediately

COROLlaRY 1. $u_{0}^{(r)}, \ldots, u_{m-1}^{(r)}$ are the imaginary parts of $f^{(r)}$ for $r \geq d$ if and only if

$$
f\left(\left[\mathbf{X}_{1}\right], \ldots,\left[\mathbf{X}_{n}\right]\right)=u_{0}+\xi u_{1}+\cdots+\xi^{m-1} u_{m-1} .
$$

We call this representation the imaginary decomposition of $f$ relative to $\xi$, and the power series $u_{0}, \ldots, u_{m-1}$ the imaginary parts of $f$.

Similarly to Lemma 3.5 in [1] we obtain a version of that lemma for power series.

LEMMA 3. $\left(u_{0}, \ldots, u_{m-1}\right)$ is a $\xi$-sequence if and only if $\left(u_{0}^{(r)}, \ldots, u_{m-1}^{(r)}\right)$ is a $\xi$-sequence for $r \geq d$.

Now we show the following generalizations of Theorems 1 and 2 .

Proposition 4. Under the assumptions of Theorem 1 on $K$ and $L$, if $u_{0}, \ldots, u_{m-1} \in K[[\mathbf{X}]]$ are power series, then the following two conditions are equivalent:

(i) $\left(u_{0}, \ldots, u_{m-1}\right)$ is a $\xi$-sequence.

(ii) There exists $f \in L[[Z]]$ such that $u_{0}, \ldots, u_{m-1}$ are the imaginary parts of $f$.

Proof. By Lemma 3 and Theorem $1,\left(u_{0}, \ldots, u_{m-1}\right)$ is a $\xi$-sequence if and only if there exist $f^{(d)}, f^{(d+1)}, \ldots \in L[Z]$ such that $u_{0}^{(r)}, \ldots, u_{m-1}^{(r)}$ are the imaginary parts of $f^{(r)}$ for $r \geq d$. By Corollary 1 this is equivalent to 
the fact that $u_{0}, \ldots, u_{m-1}$ are the imaginary parts of $f:=\sum_{r=d}^{\infty} f^{(r)}$. Thus, the proof is finished.

Proposition 5. Under the assumptions of Theorem 1 on $K$ and $L$, if the power series $u_{0}, \ldots, u_{m-1}$ are the imaginary parts of $f \in L\left[\left[Z_{1}, \ldots, Z_{n}\right]\right] \backslash\{0\}$, then $\operatorname{gcd}\left(u_{0}, \ldots, u_{m-1}\right)=1$.

Proof. If $u_{0}, \ldots, u_{m-1}$ have a common divisor in $K\left[\left[\mathbf{X}_{1}, \ldots, \mathbf{X}_{n}\right]\right]$ of positive order, then by Corollary $1, u_{0}^{(d)}, \ldots, u_{m-1}^{(d)}$ are the imaginary parts of $f^{(d)}$ and they have a common divisor in $K\left[\mathbf{X}_{1}, \ldots, \mathbf{X}_{n}\right]$ of positive degree. This contradicts Theorem 2 and ends the proof.

Analogously we obtain the following generalization of Theorem 3.

Proposition 6. Under the assumptions of Theorem 3 on $K$ and $L$, if the power series $u_{0}, \ldots, u_{m-1}$ are the imaginary parts of $f \in L\left[\left[Z_{1}, \ldots, Z_{n}\right]\right] \backslash\{0\}$, then $\operatorname{gcd}\left(u_{0}, \ldots, u_{m-1}\right)=1$.

Acknowledgments. I would like to thank the anonymous referee for his remarks improving the paper, and Professors Andrzej Schinzel and Stanisław Spodzieja for their valuable comments and advice. I am also grateful to my colleague Krzysztof Kamiński for pointing out a few mistakes in the paper.

\section{References}

[1] A. Nowicki and S. Spodzieja, Polynomial imaginary decompositions for finite extensions of fields of characteristic zero, Bull. Polish Acad. Sci. Math. 51 (2003), 157-168.

[2] A. Schinzel, Polynomials with Special Regard to Reducibility, Cambridge Univ. Press, Cambridge, 2000.

Adam Grygiel

Faculty of Mathematics and Computer Science

University of Łódź

Banacha 22

90-238 Łódź, Poland

E-mail: adamgry@op.pl

Received March 15, 2008;

received in final form March 21, 2008 